See discussions, stats, and author profiles for this publication at: https://www.researchgate.net/publication/282246268

\title{
Earprint recognition based on an ensemble of global and local features
}

Conference Paper · September 2015

DOI: 10.1109/CCST.2015.7389691

\section{CITATIONS}

4

4 authors:

(2)

Aythami Morales

Universidad Autónoma de Madrid

90 PUBLICATIONS 884 CITATIONS

SEE PROFILE

Gloria Llinas-Sanchez

Universidad de Las Palmas de Gran Canaria

1 PUBLICATION 4 CITATIONS

SEE PROFILE
234

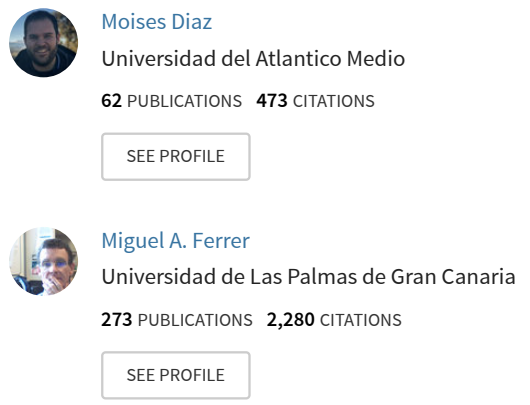

Some of the authors of this publication are also working on these related projects:

Application of the Lognormal Model to the Vocal Tract Movement View project

Signatures \& robotics View project 


\title{
Earprint recognition based on an ensemble of global and local features
}

\author{
Aythami Morales ${ }^{1}$, Moises Diaz ${ }^{2}$, Gloria Llinas-Sanchez ${ }^{2}$, Miguel A. Ferrer ${ }^{2}$ \\ ${ }^{1}$ ATVS - Departamento de Tecnología Electrónica y de las Comunicaciones, EPS, UAM, C $\backslash$ Francisco Tomás y \\ Valiente, 11, 28049 Madrid, Spain \\ ${ }^{2}$ IDeTIC - Universidad de Las Palmas de Gran Canaria, Campus de Tafira s/n, E35017, Las Palmas de Gran Canaria, \\ Spain
}

aythami.morales@uam.es,mdiaz@idetic.eu,glorial183@gmail.com,miguelangel.ferrer@ulpgc.es

\begin{abstract}
The earmarks are usual evidences in many real criminal investigations. The earprint appears for example when a criminal tries to listen through a window or a door before entering, and the methods to make it visible are similar to those used in latent fingerprint lifting. However, its acceptance as evidence in real prosecutions still raises doubts. Although it is well-accepted the uniqueness of the ear and its usefulness for person identification, the permanence of such discriminate ability in earprints is not obvious. Although the earprints do not have a powerful distinctiveness information, they are useful in a bag of evidences, being a promising soft biometric. This paper explores the discriminant properties of local descriptors for earprintbased automatic biometric recognition systems. The literature has focused on automatic systems based on the global aspect of the images, however scarcely studies have coped with in the wellknown discriminate ability of earprint local characteristics. The experiments using more than 6000 images from 1200 people suggest a promising performance in comparison with previous existing proposals based on global features and encourage to further explore in this new soft biometric traits.
\end{abstract}

Keywords-earprint, forensics, biometric, authentication.

\section{INTRODUCTION}

The security is a wide industry and research area which involves a large number of players and technologies. Among the several topics related with security technologies, the biometric recognition systems emerge as an active area which have attracted the interest of researchers, developers and consumers. Concisely, the utility of automatic identification technologies based on biometric traits in forensic scenarios is well-accepted both for research and industry communities. Although the fingerprint is probably the most popular biometric, other traits such as palmprints [3] or earprints appear usually in criminal investigations.

The utility of automatic identification technologies based on biometric traits in forensic scenarios is well-accepted both for research and industry communities [1]. Although the fingerprint [2] is probably the most popular biometric, other traits such as palmprints [3] or earprints [4] appear usually in criminal investigations.

The earmarks are usual evidences in many real criminal investigations [5]. The earprint appears for example when a criminal tries to listen through a window or a door before entering, and the methods to make it visible are similar to
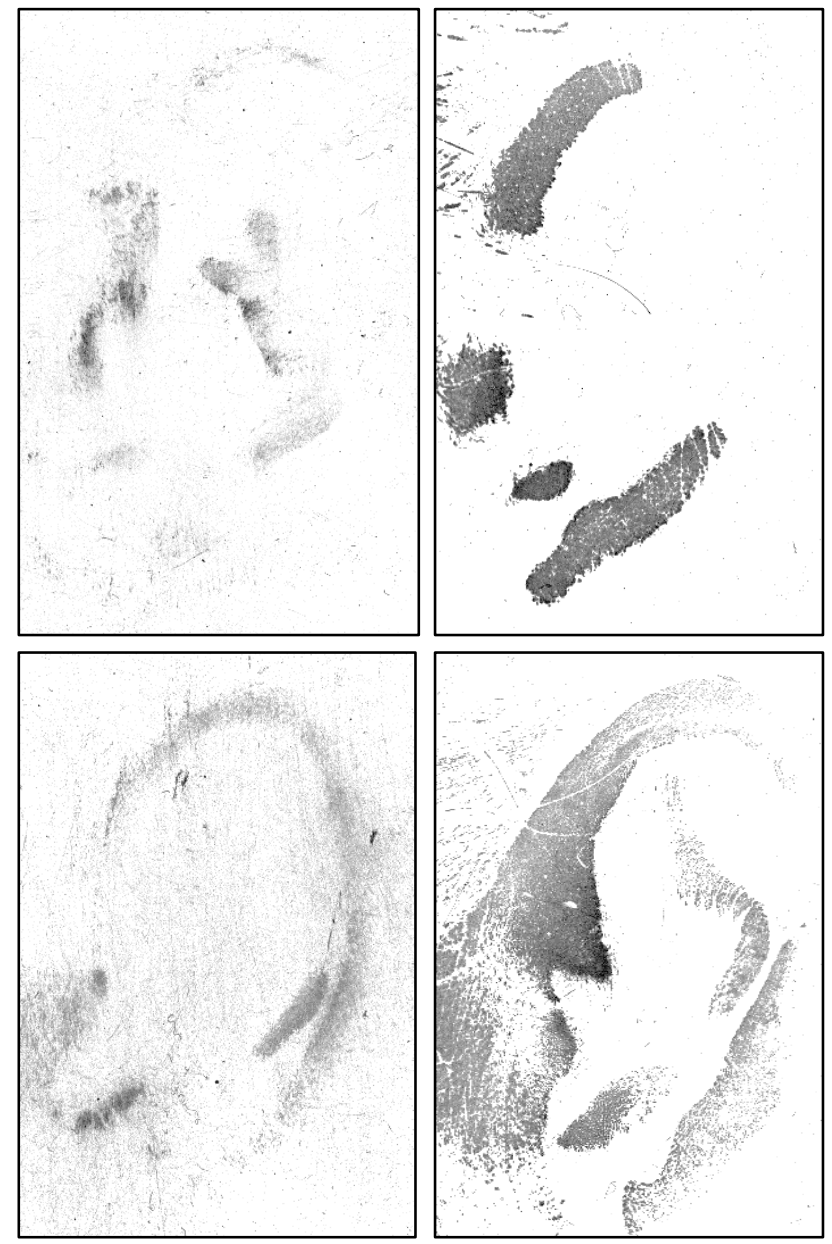

Figure 1: Examples of latent earprints (on the right) and earmarks (on the left) from different subjects from FEARDID database.

those used in latent fingerprint lifting. However, its acceptance as evidence in real prosecutions still raises doubts [6]. Although it is well accepted the uniqueness of the ear [7][8] and its usefulness for person identification, the permanence of such discriminate ability in earprints is not obvious. The earprints are defined as the marks produced by the contact between the ear and any surface.

The earprint has been used by Forensics Experts and law 


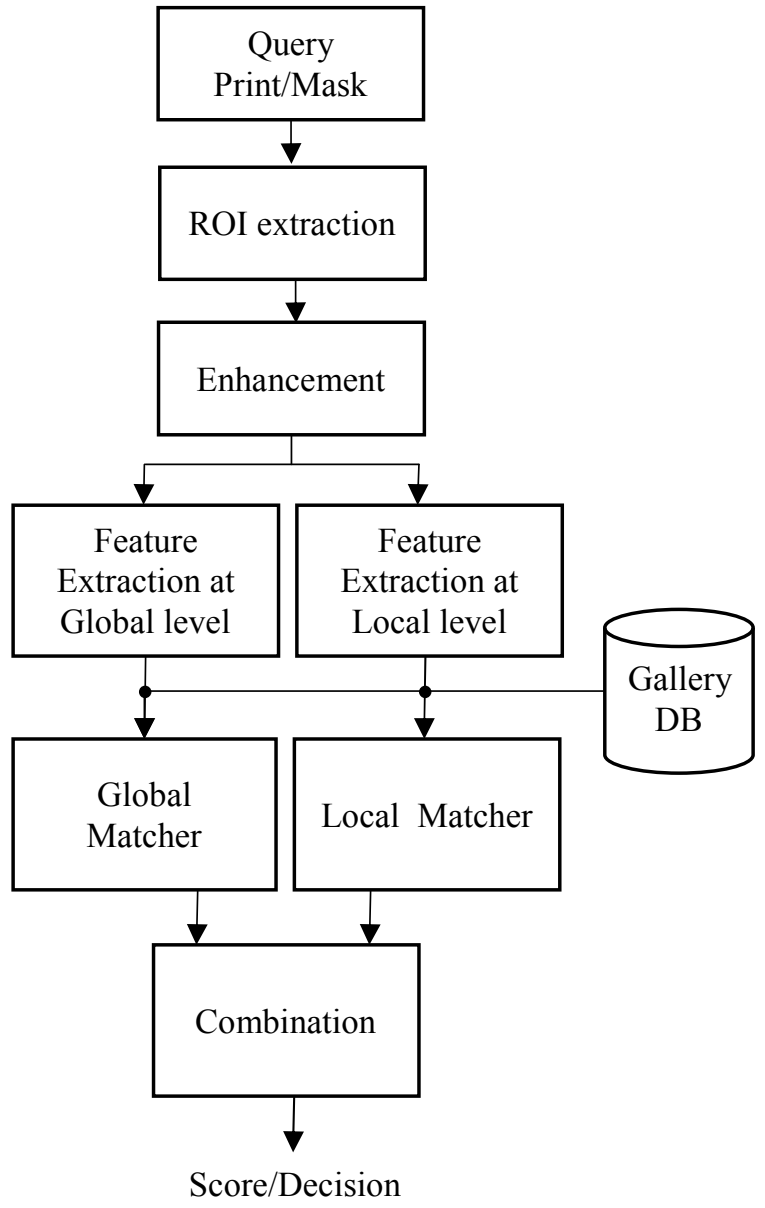

Figure 2: Block diagram of the proposed earprint identification system.

enforcements but it has attracted scarce attention of the biometric research community. The earprint is interesting for the biometric community because of: i) the pattern recognition challenges associated to low quality, missing information and large nonlinear distortions due to the flexible nature of the cartilage tissue, see Fig. 1; and ii) the social interest related to the use of earprints as evidences in real prosecutions.

The literature about earprint identification is scarce [5][9][10][11][12][13] and only few papers evaluate end-toend approaches [5][10]. The publication of the FEARID database [10] could be considered one of the most important contributions in recent years which allows to propose novel feature extraction and matching algorithms and compare it with existing ones using a common benchmark. Alberik et al. proposed in [10] a semi-automatic verification/identification system based on geometrical characteristics and manually annotation of anatomical characteristics of the earprints. In [5], Junod et al. proposed a novel semi-automatic recognition system based on image alignment and 2D Pearson-Bravais correlation coefficients. The performances achieved by both systems [5][10] were promising when images with good quality were identified. However, the lowest quality images were removed from the experiments and the performance of earprint recognition systems with low quality images is still an open challenge.

The literature on automatic earprint identification is the source for two questions raised in this paper: a) How useful are the local descriptors for automatic earprint recognition? and b) What is the performance of automatic earprint recognition approaches with low quality images?

This paper attempts to address the above challenges and analyses the performance of local descriptors for earprint recognition. The analysis of the discriminate ability of the local features of earprints can be considered as a first step (a baseline) prior to the forensics applications related to likelihood ratio and identification approaches [14]. The proposal is evaluated on a public database and the experimental results are compared with previous proposals. Our experiments suggest a promising discriminate ability of local features and its complementarity with global features for automatic earprint recognition even in the lowest quality images. The rest of the paper includes the description of the features extraction method, the experimental protocol, results and conclusions.

This was, we propose an earprint recognition system based on the ensemble of both local and global features. The proposal is evaluated on a public database and the experimental results are compared with previous proposals. The results show the effectiveness of the proposal and encourage to the research community to explore this biometric trait for further performance improvements.

The rest of the paper is organized as follow: Section 2 describes the proposed identification/recognition scheme; Section 3 deals with the database, experiments and results. Finally Section 4 draws some conclusions.

\section{METHODOLOGY}

On the one hand, the state-of-the-art of earprint identification has been traditionally focused on features characterizing the whole image (also called global features). On the other hand, the Forensic Experts Evaluations involve such global comparisons but also a carefully and deeper analysis of the local similarities between the prints. Using both global and local features is the key to further improve the automatic earprint verifiers. Global features include the greater amount of information as well as fast matching techniques. Local features are more robust to distortions and missing regions. While global features has been studied for automatic earprint recognition, to the best of our knowledge the local features have not attracted the interest of researchers yet. In this paper we propose a multialgorithm scheme based on a combination at score level of global and local features to further improve the performance of earprint recognition systems, see Fig. 2. We adopt the Semi Lights-Out methodology proposed in [5][10]. This methodology is common in Forensic Expert Evaluations and it includes a first stage in which the Expert realizes a manual evaluation (ROI 
selection in our case).

Once a query earprint is introduced in the system, the global and local features are independently extracted. The matching between the query features and the gallery features (obtained from the previously enrolled earprints) is also evaluated for both kind of features. It means that for each query earprint, two similarity scores (global and local) will be obtained. The final score or decision is worked out after the combination of both local and global similarity measures. The different blocks of the proposed diagram are presented in next sections.

\section{A. Preprocessing}

The preprocessing is realized with the aim of extract the ROI and enhance the image quality. The ROI coordinates are provided with the database (see next Section 3) and it was manually extracted by Forensics Experts according the method proposed in [5][10]. The ROI is extracted multiplying the binary mask by the earprint grayscale image. The enhancement algorithm is divided into two steps: i) an Adaptive Histogram Equalization which is applied to the ROI image in order to enhance the contrast of the regions with lowest quality; and ii) a denoising filter is applied to enhance the quality of the pattern as was proposed in [10]. The denoising filtering is defined as a $3 \times 3$ convolution according to the filter (see [10] for details):

$$
H_{d}=\frac{1}{120}\left(\begin{array}{lll}
12 & 14 & 12 \\
14 & 16 & 14 \\
12 & 14 & 12
\end{array}\right)
$$

\section{B. Global Feature Approach}

The shape of the earprint can be used to distinguish people. The most popular earprint recognition approaches are based on matching binary or grey masks obtained from the whole earprint shape. This work analyzes the performance of the algorithm proposed in [5] and its complementarity with local feature approaches. Briefly, the method can be divided into two steps (see [5] for more details):

- Creation of the earprint model: The gallery earprints are aligned using the registration algorithm proposed in [16]. All the aligned images are binarized by the Otsu method [17] which is focused on the minimization of the intraclass variance. Then, a superimposition image is worked out as a combination of the aligned earprints by adding their binarized versions.

- Comparison between the model and the query earprint: Firstly, the query earprint is aligned with the model. Once binarized, the 2D Pearson-Bravais correlation coefficient is computed using both the superimposition model and the aligned query earprint. The final score is a measure between -1 and 1 which characterizes the linear relationship between the model and the query earprint.

The main problem of these global appearance approaches is their lack of robustness in presence of incomplete or partial images (not unusual in forensic scenarios). A matching based on global image comparison can be seriously affected when the images show partial or occluded information. Is in this context where local features can be useful to deal with partial prints.

\section{Local Feature Approach}

\section{C.1. Local Feature Matching}

Local descriptors have emerged recently as a way to improve feature extraction methods in the presence of distortions such as scale, rotation, translation and occlusion. Algorithms to detect the most discriminant regions on the images have shown efficient results in many computer vision applications. The Scale Invariant Feature Transform (SIFT) algorithm is a popular method which provides a high competitive pattern recognition performance. The SIFT was originally proposed in [18] and it was already studied for CCD ear imaging biometrics in [19]. The feature extraction method can be divided into five steps:

i) Scale-space extrema detection: The Difference of Gaussian function is calculated in order to identify potential regions that show characteristics invariant to scale and rotation. The earprint image is transformed to the filtered image by the convolution $L(x, y, k \sigma)=G(x, y, k \sigma) * I(x, y)$, where $I(x, y)$ is the input image and $G(x, y, \sigma)$ is a Gaussian function with bandwidth $\sigma$ and constant multiplicative factor $k$. The Difference-of-Gaussian function is defined as:

$$
\begin{gathered}
D(x, y, \sigma)=(G(x, y, k \sigma)-G(x, y, \sigma)) * I(x, y) \\
=L(x, y, k \sigma)-L(x, y, \sigma)
\end{gathered}
$$

ii) Keypoint localization: The local maxima and minima of $D(x, y, \sigma)$ is evaluated by comparing it to its 16-neighbors. A candidate point is selected only if it is larger or smaller than all its neighbors. The candidate points with lowest contrast are rejected after performing a detailed fit to the nearby data for location, scale, and ratio of principal curvatures in the pattern. Interpolation is done using the quadratic Taylor expansion of the Difference-of-Gaussian scale-space function $D(x, y, \sigma)$ with the candidate keypointat the origin as:

$$
D(x)=D+\frac{\partial D^{T}}{\partial x}+\frac{1}{2} x^{T} \frac{\partial^{2} D^{T}}{\partial x^{2}} x
$$

being $D$ and its derivatives evaluated at the candidate keypoint and $x=(x, y, \sigma)$ the offset from this point.

iii) Orientation assignment: Each keypoint is represented by 16 orientations obtained from the local image gradient directions. For a filtered image sample $L(x, y)$ at scale $\sigma$, the gradient magnitude $m(x, y)$, and orientation, $\theta(x, y)$, are processed using pixel differences:

$$
\begin{gathered}
m(x, y)=\sqrt{\begin{array}{l}
(L(x+1, y)-L(x-1, y))^{2}+ \\
+(L(x, y+1)-L(x, y-1))^{2}
\end{array}} \\
\theta(x, y)=\tan ^{-1}\left(\frac{L(x, y+1)-L(x, y-1)}{L(x+1, y)-L(x-1, y)}\right)
\end{gathered}
$$

iv) Keypoint descriptor: Local gradients are measured at the selected scale around each keypoint. The keypoint will be defined by a descriptors vector $\left\{d_{i}\right\}_{i=1}^{M}$ with $M$ descriptors.

v) Matching: The score matching between the gallery descriptors $\left\{d_{i}^{g}\right\}_{i=1}^{M}$ and the query descriptors $\left\{d_{i}^{q}\right\}_{i=1}^{N}$ is defined by the Euclidean distances between them. Once the 


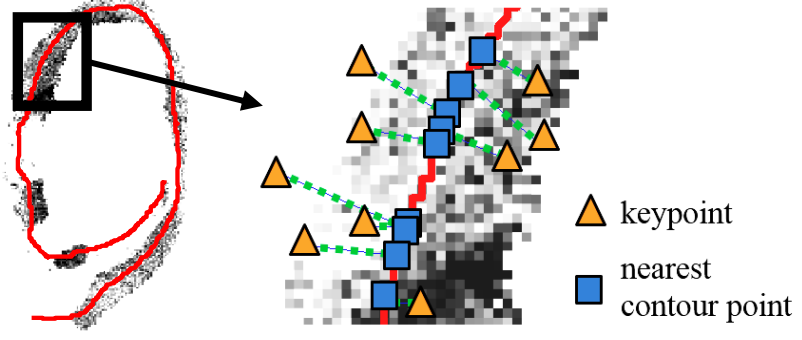

Figure 3: Earprint and contour sequence (on the left) and nearest contour point alignment for each SIFT-keypoint (on the right).

distances for all keypoints are evaluated, we consider if there is a match between keypoints when the distance between the descriptors is less than 1.5 [18].

The maximum number of matched descriptors between the query template and the gallery templates is used as a classification score or similarity measure. This method produces an elevate number of false positives and next section proposes a validation technique based on the spatial location of the matches and the contour sequence of the earprints.

\section{C.2. Validation of Local Matches}

Forensic Expert Evaluation is usually based on a set of evidences instead of only one measure. The global aspect as well as the local details are rather important in order to obtain a reliable identification. The information has a context which should be used to improve the matching performance. For example, the position of two matched features at local level could be used to discard false positives. Human experts realize these contextual evaluations intuitively but machine learning algorithms must be trained for that.

Inspired by the extended features employed in latent fingerprint recognition [2], we propose to introduce the contour sequence of the earprint as an extended feature to determine the spatial-context of two matched descriptors. The earprint contour was manually determined by Forensic Experts and it was provided with the database (described in next section).

Assuming that: i) the earprint can be divided into different sections according to the contour sequence; ii) the earprint sequence have different length and shape for each earprint; iii) two SIFT-keypoints will be correctly matched if they are located in similar sections. We propose a validation of the SIFT matches based on the locations of the keypoints and the spatial information of the contour sequence. Let $\left(x_{i}^{k}, y_{i}^{k}\right)$ be the Cartesian coordinates of each of the $M$ keypoints on the earprint and $\left(x_{j}^{c}, y_{j}^{c}\right)$ the Cartesian coordinates of the contour with $N$ concatenate points. Each keypoint is associate with its nearest position (see Fig. 3) in the contour sequence as:

$$
p_{i}=\underset{j=1, \ldots N}{\arg \min } \sqrt{\left(x_{i}^{k}-x_{j}^{c}\right)^{2}+\left(y_{i}^{k}-y_{j}^{c}\right)^{2}}, 1 \leq i \leq M
$$

A match between a gallery keypoint $A$ and a query keypoint $B$ is validated only if the distance between the two matched
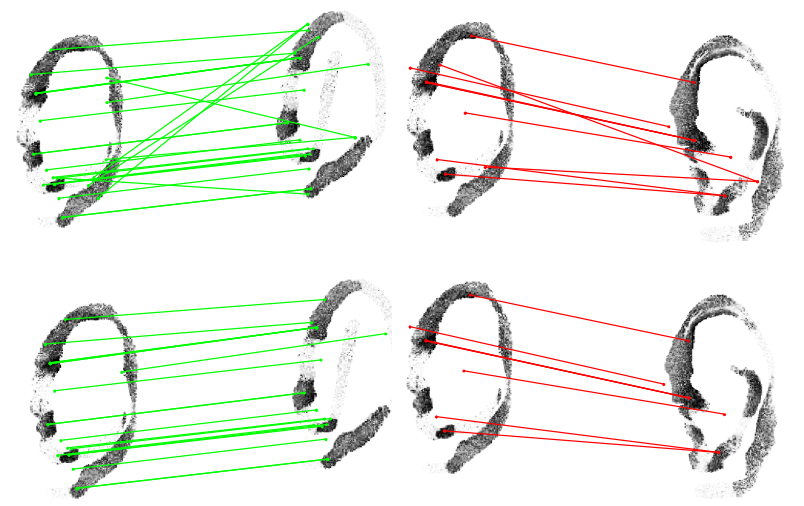

Figure 4: Earprint matching before (on top) and after (on down) the validation based on the contour sequence for a genuine comparison (on the left) and impostor comparison (on the right)

keypoints (position in the contour sequence) is lower than a threshold $\left|p_{A}-p_{B}\right|<$ threshold. In our experiments the threshold is fixed for all users in 600 .

The hypothesis is that earprints from different owners will produce more random matches than those from the same person. The proposed validation is used to reduce the number of false matches, see Fig. 4.

\section{Combination of Global and Local Approaches}

The scores obtained by the Global and the Local Extended feature approaches are normalized using the $\mathrm{min} / \mathrm{max}$ technique [20]. It allows to normalize the ranges of the classification score to [0-1] values. Finally, the normalized scores are combined according to the mean rule.

\section{EXPERIMENTS}

The experiments included in this paper are made to explore the convenience of the proposed scheme. Our experiments include a public database and comparisons with previous state-of-the-art approaches.

\section{A. Evaluation Methodology}

The evaluation methodology is supported by the database and the experimental protocols. The database used to validate the proposal is the FEARID database [10]. The database consists of 7364 prints of 1229 donors acquired by three different forensic laboratories. All donors provided three left and three right earprints. The prints were lifted according the methodology detailed in [21]. The database includes the earprint as well as the ROI (binary masks) and the contour sequences. The database also includes 216 marks which simulate realistic conditions similar to those observed in real crime scenes. The marks usually show a lowest quality than the earprints and it is considered a greater challenge.

The experiments done in this paper discarded the incomplete sets of earprints in the database (sets with less than 3 earprints). The total number of complete sets in the database is 2259 for a total number of earprints of $2259 \times 3=6777$. It is important to note that we did not discard any earprint in the 
TABLE I. Verification performance in terms of EER (\%) and quality evaluation for the FEARID database

\begin{tabular}{|c|c|c|c|c|}
\hline \multicolumn{5}{|c|}{ Print to Print Experiment } \\
\hline \multirow{2}{*}{ Features } & \multirow{2}{*}{ All } & \multicolumn{3}{|c|}{ Quality } \\
\hline & & Good & Medium & $\mathrm{Bad}$ \\
\hline Local & 3.54 & 0.001 & 0.31 & 14.26 \\
\hline Local + Extended & 2.62 & 0.001 & 0.17 & 10.97 \\
\hline Global* $^{*}[5]$ & 3.52 & 0.001 & 0.54 & 14.83 \\
\hline Combination & 1.87 & 0 & 0.04 & 9.72 \\
\hline \multicolumn{5}{|c|}{ Mark to Print Experiment } \\
\hline & All & Good & Medium & Bad \\
\hline Local & 12.31 & 0.62 & 9.85 & 45.47 \\
\hline Local + Extended & 9.22 & 0.22 & 6.15 & 36.24 \\
\hline Global* [5] & 10.54 & 0.12 & 7.70 & 47.10 \\
\hline Combination & 7.41 & 0.03 & 3.46 & 34.94 \\
\hline
\end{tabular}

*Our implementation of the algorithm proposed in [5].

database because of low quality. Removing low quality images is usual in the state-of-the-art of earprint identification [5][10] and such quality evaluation is made manually by Forensic Experts without any detail/reference about the removed images. We try to ascertain the real performance of the earprint biometric recognition system and therefore, earprints with different qualities will be evaluated. This way, although the experiments include a more realistic scenario, we aware that the inclusion of low quality samples decreases the overall performance. For that reason, the earprints are classified manually according the quality in: good $(62 \%$ of the prints), medium ( $29 \%$ of the prints) and bad ( $9 \%$ of the prints). We add the experiment with all the images to ensure a fully repeatable protocol for further research by the scientific community.

To ascertain the performance of the proposed system, the experiments include the evaluation of the verification and identification scenarios as it is common in the earprint literature. The experiments are divided into two types of comparisons:

- Print to Print experiment: the experimental protocol is done according leave-one-out technique using two earprints as gallery set and the other one as query set for each user.

- Mark to Print experiments: for each user, the three available earprints are included as gallery set and the available marks are used as query samples. This is a more challenging scenario because of the low quality of the marks which simulate real cases.

\section{B. Verification Performance}

Tables I shows the performance of the experiments in terms of Equal Error Rate for all approaches: Local features (without the matches validation based on the contour sequence), Local features + Extended (local features with validation of matches based on the contour sequence), Global features and the combination of Global and Local + Extended features.

The results show how the combination of both local and
TABLE II. Identification performance in terms of Rank-n (\%) performances for the FEARID database

\begin{tabular}{|l|c|c|c|c|}
\hline \multicolumn{5}{|c|}{ Print to Print Experiment } \\
\hline \multicolumn{1}{|c|}{ Features } & Rank-1 & Rank-5 & Rank-20 & Rank-50 \\
\hline Local & 83.7 & 90.5 & 94.9 & 96.5 \\
\hline Local + Extended & 88.1 & 94.5 & 96.7 & 97.8 \\
\hline Global* [5] & 92.6 & 94.9 & 97.4 & 98.4 \\
\hline Combination & 91.3 & 96.0 & 97.8 & 98.5 \\
\hline \multicolumn{5}{|c|}{ Mark to Print Experiment } \\
\hline Local & Rank-1 & Rank-5 & Rank-20 & Rank-50 \\
\hline Local + Extended & 51.5 & 60.8 & 73.2 & 79.4 \\
\hline Global* [5] & 62.9 & 68.0 & 81.7 & 86.3 \\
\hline Combination & 60.9 & 78.7 & 85.8 & 90.3 \\
\hline *Our implementation of the algorithm proposed in [5].
\end{tabular}

global features outperforms the individual performances. The obtained results are competitive in comparison with previous approaches and the experiments show that the quality has a high impact on the results. Note that performance cannot be directly compared to the previous works because of lowest quality images were discarded to obtain the performance in [5][10] and such quality evaluation is not publicly available. The results reported in [5][10] range between 2.31-9.3\% for the Mark to Print experiment and $0.51-3.9 \%$ for the Print to Print experiment. Concerning the differences among the features approaches, the local features outperform the global features in this recognition scenario. Our results suggest that the key to improve the performance of earprint verification systems is dealing with lowest quality images. According to the Mark toPrint experiments, the drop of performance is evident and the EER is near the triple of the EER obtained for the Print to Print experiments. However, the good quality prints show a promising performance and suggest that a correct recognition is possible in such conditions. The improvement obtained by the combined approach is clear and suggest the complementarity of both global and local features.

\section{Identification Performance}

While recognition is a $1: 1$ comparison, the identification is a 1: $n$ comparison in which $n$ in the number of identities in the database. Table II shows the performance (Rank-n) of the different feature approaches.

The identification experiment shows a competitive rank-n accuracy for the Print to Print experiment but the performance is moderate for the Mark to Print scenario. Note that in identification mode, competitiveness of the different feature approaches changes regarding to the recognition mode. In this case, global features show more competitive performances and the combination produces a scarce improvement for rank- 5 in advance. The divergence between recognition and identification performances was studied in [22]. A good recognition performance does not necessarily means a good identification performance. The match score distributions 
determine the different performances and the narrow impostor score distribution of the local feature approach can be the reason of the poor identification results.

\section{CONCLUSIONS}

This paper analyses the performance of local descriptors for automatic earprint recognition. The proposal includes the use of a spatial validation of local features based on the ear contour and results suggest the competitive performance of the proposed procedure. Two questions were raised in the first section of this paper: How useful are local descriptors for automatic earprint recognition? What is the performance of automatic earprint recognition with low quality images? The local features show competitive performances in verification experiments and combination with global features outperform the state-of-the-art systems. The results suggest the complementarity of the global and local features for the recognition experiments and encourage us to advance the study of new improvements. The results highlight the impact of the quality on the performance of automatic earprint recognition systems and point out that there is a large room for improvements in samples with lowest quality.

\section{ACKNOWLEDGMENT}

A.M. is supported by a post-doctoral Juan de la Cierva contract by the Spanish MECD (JCI-2012-12357). M.D.-C. is supported by a Ph.D. fellowship from the ULPGC. This work has been partially supported by project BioSint (TEC201238630).

\section{REFERENCES}

[1] D. Dessimoz, C. Champod, "Linkages between biometrics and forensic science", Anil K. Jain (Ed.) et al., Handbook of Biometrics, Springer, Berlin (2008).

[2] A. K. Jain and J. Feng, "Latent fingerprint matching," IEEE Trans. On Pattern Anal. Mach. Intell. 33(1): 88-100, 2011.

[3] A. K. Jain, J. Feng, "Latent palmprint matching", IEEE Trans. on Pattern Anal. Mach. Intell. 31(6): 1032-1047, 2009.

[4] A. Iannarelli, Ear Identification (Forensic Identification Series ed.), Paramount Publishing Company, Fremont, California, 1989.

[5] S. Junod, J Pasquier. The development of an automatic recognition system for earmark and earprint comparisons. Forensic Sci. Int. 222: 170-178, 2012.

[6] State v. David Wayne Kunze, Court of Appeals of Washington, Division 2, 97 Wash. App. 832, 988 P.2d 977, 1999.

[7] C. Champod, I.W. Evett, B. Kuchler, Earmarks as evidence: a critical review, J. Forensic Sci. 46 (6): 1275-1284, 2001.

[8] L. Meijerman, S. Sholl, F. De Conti, M. Giacon, C. Van der Lugt, A. Drusini, P. Vanezis, G. Maat. Exploratory study on classification and individualization of earprints. Forensic Sci. Int. 140: 91-99, 2004.

[9] G.N. Rutty, A. Abbas, D. Crossling. Could earprint identification be computerised? An illustrated proof of concept paper, Int. J. Legal Med. 119(6): 335-343, 2005.

[10] I. Alberink, A. Ruifrok, Performance of the FearID earprint identification system, Forensic Sci. Int. 166 (2-3): 145-154, 2007.

[11] L. Meijerman, A. Thean, C. Van der Lugt, R. Van Munster, G. Van Antwerpen, G. Maat. Individualization of earprints: variation in earprints of monozygotic twins, Forensic Sci. Med. Pathol. 2(1): 39-49, 2006.

[12] L. Meijerman, S. Sholl, F. De Conti, M. Giacon, C. Van der Lugt, A. Drusini, P. Vanezis, G. Maat, Exploratory study on classification and individualization of earprints, Forensic Sci. Int. 140: 91-99, 2004.

[13] Z. Johnson, Operating Procedure for the Taking of Earprints, Internal FearID Report, 2003 (online), http://forensic.to/fearid/Procedure.doc.

[14] D. Ramos, Forensic Evaluation of the Evidence Using Automatic Speaker Recognition Systems, Universidad Autonoma de Madrid, November 2007.

[15] V. N. Dvornychenko, Michael D. Garris, "Summary of NIST Latent Fingerprint Testing Workshop" NISTIR 7377, November 2006.

[16] P. Thevenaz, U.E. Ruttimann, M. Unser. A pyramid approach to subpixel registration based on intensity, IEEE Trans. Image Process. 7 (1): 27-41, 1998.

[17] N. Otsu, A threshold selection method from gray-level histograms, IEEE Trans. Syst. Man Cybern. 9 (1): 62-66, 1979.

[18] D. Lowe, Distinctive image features from scale-invariant keypoint. Int. J. Comput. Vision, 60 (2): 91-110, 2004.

[19] Aythami Morales, Miguel A. Ferrer, Moises Diaz-Cabrera, Esther Gonzalez. Analysis of local descriptors features and its robustness applied to ear recognition", 47th IEEE International Carnahan Conference on Security Technology, Medellin, 2013, pp. 171-175.

[20] A. K. Jain, K. Nandakumar, A. Ross. Score normalization in multimodal biometric systems. Pattern Recognition. (38): 2270$2285,2005$.

[21] I. Alberink, A. Ruifrok. Repeatability and reproducibility of earprint acquisition”. J. Forensic Sci. 53: 325-30, 2008.

[22] B. Decann, A. Ross, Can a poor verification system be a good identification system? A preliminary study. IEEE International Workshop on Information Forensics and Security (WIFS), Tenerife, Spain, 2012, pp. $31-36$. 